\title{
Semimagnetic Semiconductors
}

\author{
Robert R. Galazka, Warsaw
}

(Institute of Physics, Polish Academy of Sciences)

The solid state can be divided into a few large groups amongst which can be numbered the semiconductors (SC) and the magnetic materials (MM). Although these have many features in common the two groups are very different. The properties of SC are governed by the properties of mobile, delocalized charge carriers, while the properties of most $\mathrm{MM}$ arise from spin exchange interactions between localized paramagnetic ions.

Exchange interactions are usually negligible in SC, the lattice being diamagnetic; the paramagnetism of the electron gas is weak and has no significant influence on the properties of the material. Spin dependent phenomena such as the spin splitting of bands or impurity levels are important however, and have been extensively investigated as well as other effects induced by an external magnetic field. In $\mathrm{MM}$ the exchange interaction between localized paramagnetic ions is usually short-range although in metallic alloys the presence of conduction electrons can mediate a longrange interaction between magnetic ions - the Ruderman-Kittel-KasuyaYoshida mechanism. RKKY theory predicts an interaction that oscillates between ferromagnetic and antiferromagnetic, and decreases with distance. This interaction is strongly dependent on the carrier and magnetic ion concentrations and plays an important role in dilute magnetic alloys. For SC, the concentration of carriers that is typical, is too low for any significant RKKY-type of interaction to be observed.

Semimagnetic semiconductors (SM$\mathrm{SC}$ ) are new materials combining both semiconductor and magnetic properties. Their semiconductor properties are still governed by delocalized carriers but, owing to the presence of paramagnetic ions, the exchange interaction between electrons and ions can significantly modify the properties of the carriers. On the other hand, the electronic properties such as band structure or carrier concentration can influence the interaction between magnetic ions and, in turn, their magnetic properties.

From the point of view of chemical composition, SMSC are mixed crystals or solid solutions of semiconductor compounds, as e.g. CdTe, $\mathrm{HgSe}, \mathrm{PbS}, .$. in appropriate magnetic compounds like MnTe, MnSe, FeSe or MnS. Currently, the most investigated SMSC contain $\mathrm{Mn}$ as paramagnetic ion, but newly created SMSC with Fe and Eu are also the subject of intensive studies.

Properties of Carriers Affected by Exchange Interactions with lons

Replacing cations such as $\mathrm{Cd}$ or $\mathrm{Hg}$ with a paramagnetic ion such as $\mathrm{Mn}$ in the same crystallographic structure does not markedly disturb the semiconductor properties of the material. The energy gap changes (increases) but the conduction and valence bands conserve their symmetry and character as in nonmagnetic semiconductor mixed crystals. The spin momentum of $\mathrm{Mn}$ is connected with the $3 d^{5}$ shell; the energy level is about $3 \mathrm{eV}$ below the top of the valence band and has thus negligible influence on its shape or that of the bottom of the conduction band. Thus all basic semiconductor properties connected with the band symmetry and topology are the same as for a typical semiconductor and the only influence of the magnetic sub-systems on electrons comes from the spin exchange interaction between mobile carriers and localized magnetic ions. This interaction can be represented by a Heisenberg term $H_{\text {ex }}$ $=J \sigma . S$, where $\sigma$ and $S$ are the spin operators for an electron and an ion respectively and $J$ is the exchange constant ferromagnetic for electrons and antiferromagnetic for holes.

The Heisenberg term must be added to the effective mass Hamiltonian to solve the energy eigen problem for SMSC. Realising this was the turning point in understanding their properties and provided a basis for this group of alloys to be distinguished from other semiconductor mixed crystals. Since that time (about 10 years ago) SMSC have become the subject of intensive studies in Europe and the USA.

The exchange interaction depends on the ion spin and the exchange constant. The spin of $\mathrm{Mn}$ is the highest for transition metals and equals 5 Bohr magnetons, and whilst a typical value for the exchange coupling between magnetic ions $J_{d-d}$ is $10^{-3} \mathrm{eV}$, the exchange constant for electrons and holes with magnetic ions $J_{\text {sp-d }}$ is about $1 \mathrm{eV}$ in SMSC.
Fig. 1 - Conduction and valence bands quantization in external magnetic field of $\mathrm{Cd}_{0.95} \mathrm{Mn}_{0.05} \mathrm{Te}$ and CdTe. Spin splitting - very weak in CdTe is the main effect in CdMnTe. Notice almost equal splitting of heavy and light hole bands in CdMnTe. The influence of exchange interaction is visible even at $100 \mathrm{~K}$. The picture of CdTe in this range of temperature is practically the same.

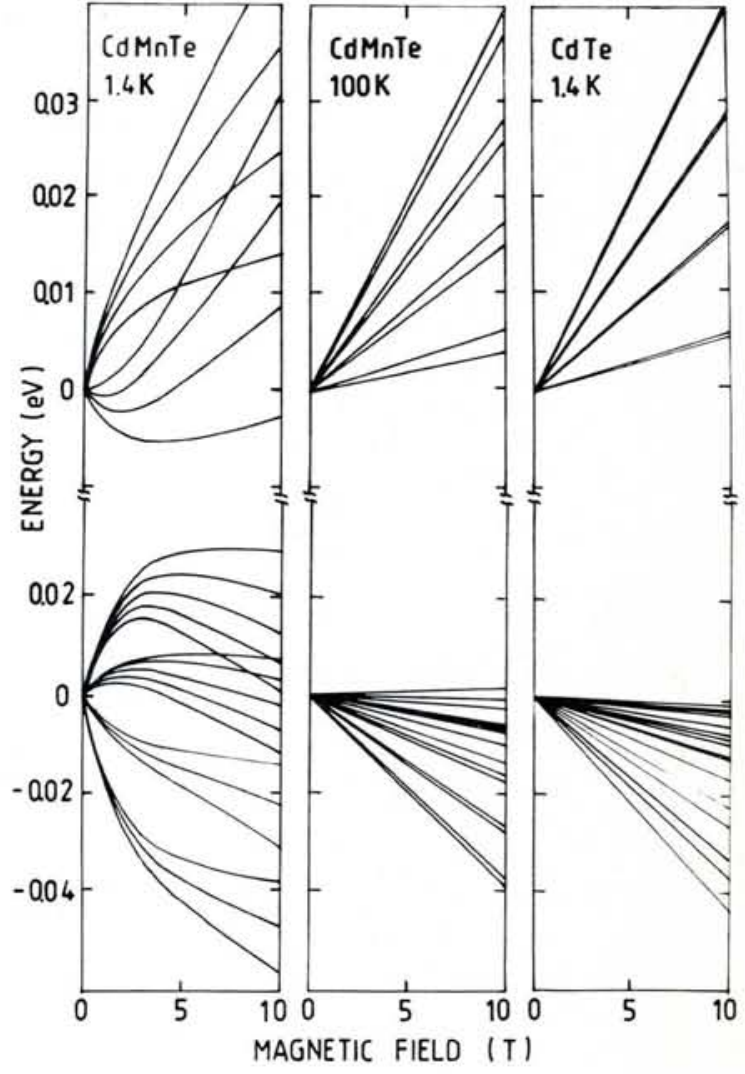


The exchange interaction is also strongly temperature and magnetic field dependent. As the macroscopic magnetization of a sample is proportional to the thermodynamic average value of the magnetic ion spin, to a good approximation we can replace the ion spin operators in the Hamiltonian by their average value, calculated or taken from measurements of the magnetization. From typical magnetic functions we can thus obtain information on the electron behaviour.

In semiconductors, an external magnetic field acts on both the orbital motion and the spin of electrons producing Landau quantization and spin splitting, to an extent dependent on the effective mass of the carriers. The exchange interaction acts on their spin only and is, in effect, mass independent. Because of this and also the large value of $J_{s p-d}$, the band structure in turn changes drastically under the influence of an external magnetic field and depends strongly on temperature. From Fig. 1 we can see that the very typical structure of the splitting of the valence bands disappears in SMSC when a magnetic field is applied. The change is accompanied by a drastic increase of hole mobility like that observed in two-dimensional structures. In SMSC such as $\mathrm{Hg}_{1-x} \mathrm{Mn}_{\mathrm{x}} \mathrm{Te}$ or $\mathrm{Hg}_{1-x} \mathrm{Mn}_{x} \mathrm{Se}$ (for $x \leq 0.07$ ) the magnetic field produces an overlap of the valence and conduction bands, an effect never observed in non-magnetic semiconductors. (For details see Ref. 2, 2nd and 3rd papers.)

Impurity levels are also influenced by exchange reactions: the ionization energy of an acceptor decreases and its wave function becomes anisotropic under the influence of a magnetic field. Both effects produce a giant negative magneto-resistance and a field-induced insulator-metal transition. Indeed all the properties susceptible of being changed by a magnetic field are very different in SMSC in comparison with ordinary SC - the Faraday effect, Shubnikov-de Haas oscillations, magneto-optical properties, luminescence; these are all different and are also strongly temperature dependent.

In the absence of a magnetic field, the average value of the ion spins is zero, the magnetization is zero too, and SMSC should behave as typical non-magnetic semiconductors. Whereas this is true for delocalized band electrons, when an electron is localized around an impurity, a bound magnetic polaron can be observed. This concept was first introduced to explain the behaviour of magnetic semiconductors (Eu chalcogenides) and then later was applied also to SMSC. A localized electron can produce a spontaneous magnetization within the range of its wave function via the exchange interaction. Local ferromagnetic ordering in that range produces a Stoke's shift in spin-flip Raman scattering even in the absence of an external magnetic field and other related phenomena. Local fluctuations of magnetization and a detailed knowledge of electronic states are essential for a theoretical description of this effect. The bound magnetic polaron is a subtle example of the feedback between electronic and magnetic sub-systems present in SMSC.

\section{Ordering of Magnetic lons}

Magnetic properties of SMSC connected with the exchange interaction between paramagnetic ions are similar to those of other dilute magnetic materials. The broad range of possible magnetic ion concentrations (up to $x=$ 0.80 in $\mathrm{Zn}_{1-x} \mathrm{Mn}_{x} \mathrm{Te}$ and $x=0.70$ for $\mathrm{Cd}_{1-x} \mathrm{Mn}_{x} \mathrm{Te}$ ) allows us to observe the evolution of magnetic interactions in the same crystallographic structure as a function of magnetic ion concentration. A continuous transition from diamagnetic behaviour (for $x \leq 0.005$ ) of the host semiconductor, to paramagnetism, spin-glass (for $x \geq 0.02$ ) and finally antiferromagnetic order has been observed. Type III antiferromagnetic ordering is observed for $x>0.6$ indicating that the magnetic elementary cell contains two elementary cells of the cation sublattice. From a crystallographic point of view the magnetic sub-system is disordered; the distribution of magnetic atoms on the cation sub-lattice (f.c.c. or h.c.p.) is random.

A spin-glass like state is observed in SMSC in the broadest range of paramagnetic ion concentrations $10.02 \leq x \leq$ $0.060)$ and above the percolation threshold this is not canonical. Indeed the temperature dependence of the magnetic susceptibility below and above the transition temperature and their dynamic properties at low and high magnetic fields suggest rather the existence of two spin-glass phases arising from the competition between spin-glass and clustering behaviour. Below the percolation threshold ( $x \leq 0.17$ for f.c.c. lattice) the mechanism responsible for a spinglass phase is even less clear although, for such a dilute system it must be longrange to produce freezing of the spins.

The antiferromagnetic order has been inferred from specific heat and magnetic susceptibility measurements, and additional information concerning this phase has come from neutron diffraction stu-

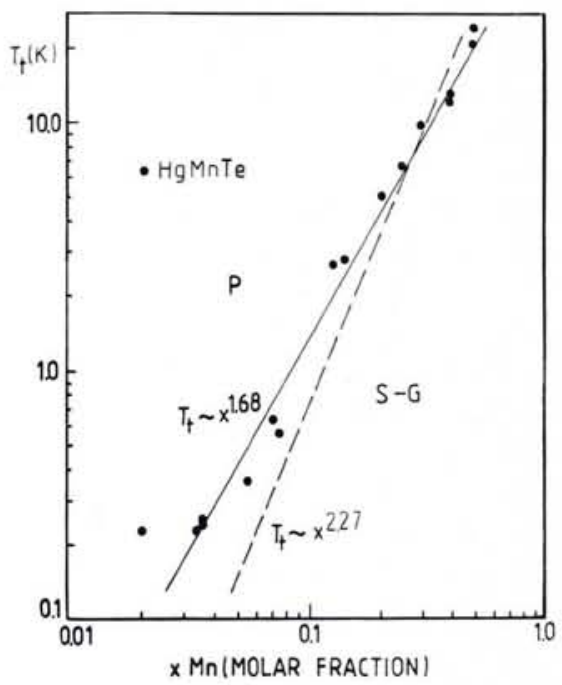

Fig. 2 - Magnetic phase diagram of the majority of SMSC with Mn. Broken line represents wide gap SMSC as ZnMnSe, ZnMnTe, CdMnTe and CdMnSe (Ref. 3); solid line was drawn through the experimental points collected in Ref. 2, paper 4 for zero gap HgMnTe. For $x>0.20 \mathrm{HgMnTe}$ becomes the wide gap SMSC and experimental points are close to the other wide gap materials. Different slopes of the lines indicate the band structure influence on the spin-glass transition temperature.

dies: only a certain fraction of the total number of magnetic ions (e.g. about $50 \%$ for CdMnTe, $x=0.65$ ) is well ordered, the rest remain in a disordered spinglass phase. Thus a mixed phase (antiferromagnetic and spin-glass coexisting together) rather than truly antiferromagnetic phase is observed.

Despite the variety of semiconductor host compounds, many magnetic properties are common to the whole group of SMSC:

- magnetic phase diagrams are very similar;

- interaction between paramagnetic ions is antiferromagnetic for nearest and next neighbours;

- both short-range and long-range magnetic interactions are present and are important;

— different magnetic phases coexist.

\section{Effects of Carriers on Magnetic Proper- ties}

The influence of basic electronic properties such as band structure and carrier concentration on the magnetic properties of SMSC is also significant. The former is related to the position of the $3 d$ level of the paramagnetic ion with respect to the valence or conduction bands and on the hybridization of this level. Strong hybridization occurs whenever the $3 \mathrm{~d}$ level is close to a band extremum provided the symmetry of the band allows the interaction. Moreover, the ef- 
fective exchange constant $J_{d-d}$ which is proportional to $V_{h}^{4}$, where $V_{h}$ is the hybridization energy also increases. Higher values of $J_{d-d}$ are observed for sulphides and selenides than for tellurides if alloys with the same cation are considered. This is in agreement with the expected upward shift of the $3 \mathrm{~d}$ level of $\mathrm{Mn}$, from about $3 \mathrm{eV}$ below the top of the valence band for tellurides, to an energy difference close to zero for sulphides.

The next effect related to the band structure and particularly the energy gap is the Bloembergen-Rowland mechanism leading to the exponential decay $J_{d-d} \sim J_{0} \exp (-\alpha R)$ for finite gap materials and a power law decay of $J_{d-d}$ for zero gap SMSC as in HgMnTe or $\mathrm{HgMnSe}$. The exponential or power decay produces a long-range interaction with respect to short-range, delta-like interactions usually assumed for exchange mechanisms between magnetic ions. The spin-glass transition temperature is particularly sensitive to the longrange part of the exchange interactions as can be seen in Fig. 2 which gives data on both wide gap SMSC and HgMnTe. The difference in the response to changing $\mathrm{Mn}$ concentration in the zero or narrow gap HgMnTe, suggests that band structure influences spin-glass formation.

The influence of carrier concentration on magnetic properties has been extensively investigated in metallic alloys. Several IV-VI semiconductors like SnTe can exhibit quite high metallic-like carrier concentrations. In the quarternary alloy $\mathrm{PbSnMnTe}$, the concentration of holes can be varied by annealing in the range $10^{20}$ to $5 \times 10^{21} \mathrm{~cm}^{-3}$. In $\mathrm{Pb}_{0.25} \mathrm{Sn}_{0.72} \mathrm{Mn}_{0.03} \mathrm{Te}$ an abrupt transition from paramagnetic to ferromagnetic phase has been found for $p>3 \times$ $10^{20} \mathrm{~cm}^{-3}$. The Curie temperature is a function of hole concentration, but the

\section{University of São Paulo at São Carlos, São Paulo BRASIL}

\section{RESEARCH POSITIONS IN EXPERIMENTAL CONDENSED MATTER PHYSICS}

At the Physics Department of the Instituto de Física e Química de São Carlos, from the University of São Paulo, post-doctoral research positions are available.

Research connected with these positions is in the fields of Semiconductors, Non-Linear Optics and Quantum Electronics, Crystal Growth, Biophysics, NMR Imaging, Superconductivity, Electrets, Crystallography, Polymers, Glasses and Ceramics.

Candidates will be expected to carry out research in conjunction with other members of the Department and to play an active role in initiating and developing new programmes.

Applicants should have Ph.D. qualification in experimental physics; a period of relevant post-doctoral experience would be an advantage.

The duration of the contract is one to two years with an extension possible. The salary will depend on qualifications and experience and will be the same as that of equivalent staff members of the University of São Paulo.

Candidates should send a Curriculum Vitae, list of publications, patents, etc., and three letters of reference, to
Prof. Dr. Oscar Hipólito, Universidade de São Paulo Instituto de Física e Química de São Carlos
Departamento de Física e Ciência dos Materials
Caixa Postal 369, 13.560 - São Carlos - São Paulo - Brasil

$\mathrm{Mn}$ ions conserve their magnetic moment of 5 Bohr magnetons. The magnetic phase diagram for this first ferromagnetic SMSC is thus three dimensional $(T, x, \eta)$ in contrast to the two dimensional diagram $(T, x)$ usually presented for magnetic alloys. Even low concentration of spin polarized carriers when optically pumped can change the magnetization of $\mathrm{HgMnTe}$ owing to the strong exchange interaction of carriers with magnetic ions.

This overview of the research conducted in the field of SMSC indicates the number of phenomena that can be studied in a novel context as well as new effects appearing at the interface between the physics of semiconductors and magnetic materials. The mixing of states and the coupling effects between electronic and magnetic sub-systems conspire to make SMSC a fascinating field of research.
The Polish version of this article will be published in the Polish Physical Society journal Postepy Fizyki.

\section{REFERENCES}

1. Galazka R.R. and Kossut J. in: LandoltBörnstein New Series 17b, Eds. O. Madelung, M. Schulz and M. Weiss, (Springer, Berlin) 1982, p. 302. (Collected numerical data of SMSC.)

2. Recent review papers:

Mycielski J., Progr. Crystal Growth and Characterization 10 (1984) 101;

Brandt N.B. and Moshchalnikov V.V., Adv. Phys. 33 (1984) 139;

Galazka R.R., J. Crystal Growth 72 (1985) 364;

Galazka R.R., Proc. 18th ICPS, Stockholm 1986 , in press.

3. Twardowski A., Denissen C.J.M., de Jonge W.J.M., de Waele A.T.A.M., Demianiuk M. and Triboulet R., Sol. State Comm. 59 (1986) 199.

4. Mycielski A., Rigaux C., Menat M., Dietl T. and Otto M., Sol. State Comm. 50 (1984) 257.
EPS Divisions, Sections and Group Astronomy and Astrophysics Division Solar Physics Section

Atomic and Molecular Physics Division Atomic Spectroscopy Section Chemical Physics Electronic and Atomic Collisions Molecular Physics Computational Physics Group

Condensed Matter Division Liquids Section Low Temperature Physics Section Macromolecular Physics Magnetism Metal Physics Semiconductors and Insulators Surfaces and Interfaces High Energy \& Particle Physics Division Interdiv. Group on Exptl. Phys. Control Systems Interdiv. Group on Physics for Development Nuclear Physics Division Optics Division

Plasma Physics Division

Quantum Electronics Division
Europhysics News is the official journal of the European Physical Society which comprises 29 National Societies, Academies and Group, about 4000 Individual Members and 70 Associate Members. Governing bodies of EPS are the General Meeting, Council and an elected Executive Committee responsible for detailed policy. EPS promotes the collaboration of physicists throughout Europe, organising and harmonising conferences and publications, improving physics education, encouraging physics applications, awarding scholarships to sponsored schools in Erice. EPS publishes in addition to Europhys. News, Europhysics Letters lin partnership with national societies). European Journal of Physics (in partnership with The UK Inst. of Phys.), of Physics (in partnership with The UR Inst. of Phys.),
and European Conference Abstracts. Individual Members receive Europhys. News free of charge (price to insts. Sw.Fr. 90.-la), Europhys. Lett. at Sw.Fr. 70.-1a (insts. 595.-1, rebates on many other publications and on conference fees. Annual EPS membership fee for individual Members belonging to an EPS member society is: Sw.Fr. 44. - ; independent members: Sw.Fr, 132.members of a Collaborating Society: Sw.Fr. 55.-.

\section{Editor: E.N. Shaw}

Editorial Board:

A. Baratoff, F. James, M. Lehmann,

M. Mayor, J. Muller, M. Siegrist

Editorial and Advertising Office at the EPS Secretariat

Address: EUROPEAN PHYSICAL SOCIETY P. O. Box 69 , CH-1213 Petit-Lancy 2 Switzerland

Telephone: Geneva (22) 931130 Telex: 428024 eps ch Cables: europhys genève

Printed by: Pfirter frères sa $\mathrm{CH}-1213$ Petit-Lancy/Switzerland 